\title{
High-dose intravenous immunoglobulin therapy induces and maintains complete remission for polyarteritis nodosa
}

\author{
Kazu Ode ${ }^{1}$, Yoshinori Taniguchi ${ }^{1}$, Yoshitaka Kumon $^{2}$, Yoshio Terada $^{1}$ \\ 1. Department of Endocrinology, Metabolism and Nephrology, Kochi University School of Medicine, Nankoku, Japan. \\ 2. Division of Rheumatology, Chikamori Hospital, Kochi, Japan
}

Correspondence: Yoshinori Taniguchi, M.D. Address: Department of Endocrinology, Metabolism and Nephrology, Kochi Medical School, Kochi University, Kohasu, Oko-Cho, Nankoku, Kochi 783-8505, Japan. Email: taniguchiy@kochi-u.ac.jp

Received: December 12, 2013

Accepted: February 7, 2014

Online Published: February 11, 2014

DOI : $10.5430 /$ crim.v1n1p13

URL: http://dx.doi.org/10.5430/crim.v1n1p13

\begin{abstract}
We report a case of successful high-dose intravenous immunoglobulin (IVIG) use in a patient with refractory polyarteritis nodosa (PAN). Treatments with prednisolone (PSL) and various types of immunosuppressants including methotrexate (MTX) and intravenous cyclophosphamide (IVCY) were unsuccessful, and then, high-dose IVIg therapy was added. High-dose IVIG therapy improved all symptoms including high fever, arthralgia, mononeuritis multiplex and indurated erythema due to PAN. Moreover, serum inflammatory markers were also normalized. High-dose IVIG is maintaining complete remission for PAN without flare-up for additional 4 years. Therefore, high-dose IVIG therapy might be considered as a first-line therapy in patients with PAN or alternative therapy in refractory PAN.
\end{abstract}

\section{Key words}

Intravenous immunoglobulin, Polyarteritis nodosa, Refractory, Remission

\section{I ntroduction}

Polyarteritis nodosa (PAN) is a systemic necrotizing vasculitis that typically affects medium-sized muscular arteries, with occational involvement of small muscular arteries. The disease can affect any organ of the body because systemic arteries are involved. Since the clinical course of PAN shows chronic pattern and life-threatening events are frequently revealed, the long-term administration of immunosuppressants is known as the standard of care ${ }^{[1]}$. Recently, the effects of high-dose intravenous immunoglobulin (IVIG) have been described for various rheumatic disorders, including PAN ${ }^{[2-5]}$. We herein report successful high-dose IVIG use in a patient with refractory PAN.

\section{Case presentation}

A 36-year-old Japanese woman was admitted to our hospital on September 2008 with high fever, general fatigue, arthralgia, numbness of the right upper and left lower extremities and indurated erythema. Livedo reticularis and indurated erythema (see Figure 1A) were revealed in the lower extremities. Regarding laboratory data, the white blood cell count was $9200 / \mu 1$, hemoglobin level was $11.5 \mathrm{~g} / \mathrm{dl}$, and platelet count was $33.4 \times 10^{4} / \mu 1$. Erythrocyte sedimentation rate (ESR) 
was $101 \mathrm{~mm} / \mathrm{hr}$, C-reactive protein (CRP) level was $5.4 \mathrm{mg} / \mathrm{dl}$, and an elevated serum complement level (CH50, 59.4/ml) was shown. Anti-nuclear antibody was positive at 1:1280 with a centromere pattern. Serum hepatitis B surface antigen (HBsAg), anti-neutrophil cytoplasmic antibody (ANCA), anti-Parvovirus B19-IGM, Epstein-Barr virus (EBV) viral capsid antigens (VCA)-IGM, EBV early antigen-diffuse and restricted antibody (EA-DR)-IGG and EBV EA-DR-IgA were all negative. Urine analysis, renal and hepatic function tests were all within normal range. The nerve conduction study showed that the conduction velocity was lowered in the right ulnar, left tibial and sural nerves (both sensory and motor), indicating mononeuritis multiplex. An incisional skin biopsy specimen obtained from the indurated erythema of lower extremities (Figure 1A) demonstrated necrotizing arteritis with fibrinoid degeneration involving a medium-sized artery from the deep dermis to a fatty layer of the subcutaneous dermis (see Figure 1B). 3D-CT angiography showed an aneurysmal change in the celiac artery. She was diagnosed as systemic PAN based on these findings, and was initially treated with methotrexate (MTX), $8 \mathrm{mg}$ weekly, and prednisolone (PSL), $40 \mathrm{mg}$ daily, after intravenous steroid pulse therapy. Because of the poor response of erythema and mononeuritis multiplex to this treatment, six courses of intravenous cyclophosphamide (IVCY) pulse therapy, $600 \mathrm{mg} / \mathrm{m}^{2}$ at an interval of every 4 weeks, was initiated. This treatment provided neurological relief from numbness and pain, however, PAN flared up with a high fever, erythema and exacerbation of neuritis in 2009. In addition to PSL, $15 \mathrm{mg}$ daily, and MTX, $8 \mathrm{mg}$ weekly, high-dose IVIG at a dose of $0.4 \mathrm{~g} / \mathrm{kg} /$ day for five consecutive days was started. After the completion of the first course of high-dose IVIG, all symptoms of PAN including high fever, arthralgia, mononeuritis multiplex and erythema were dramatically improved. Moreover, inflammatory markers including CRP and ESR were also normalized. PSL was gradually tapered and MTX was changed to azathioprine (AZT) as a maintenance therapy after high-dose IVIG therapy. High-dose IVIG is currently maintaining a complete remission for PAN without flare-up for an additional 4 years (see Figure 2).
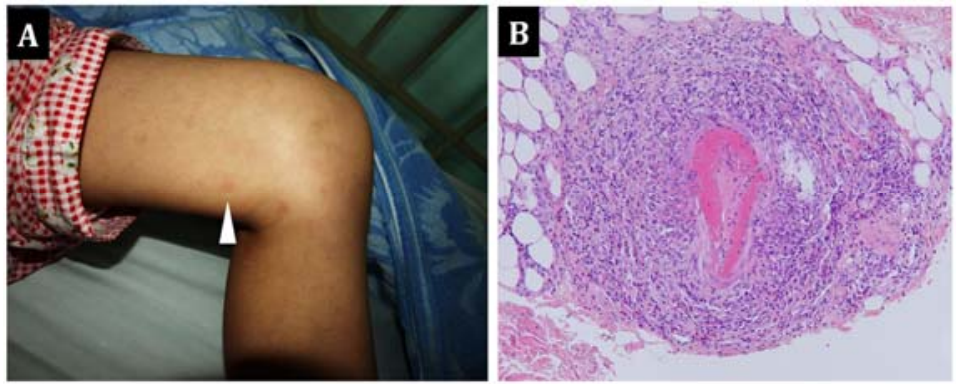

Figure 1. (A) Indurated erythema (arrow heads) and livedo reticularis were revealed at the lower extremities. (B) An incisional skin biopsy specimen obtained from indurated erythema demonstrated necrotizing arteritis with fibrinoid degeneration involving a medium sized artery from the deep dermis to a fatty layer of subcutaneous dermis. Hematoxylin-eosin stain: original magnification: 100×.

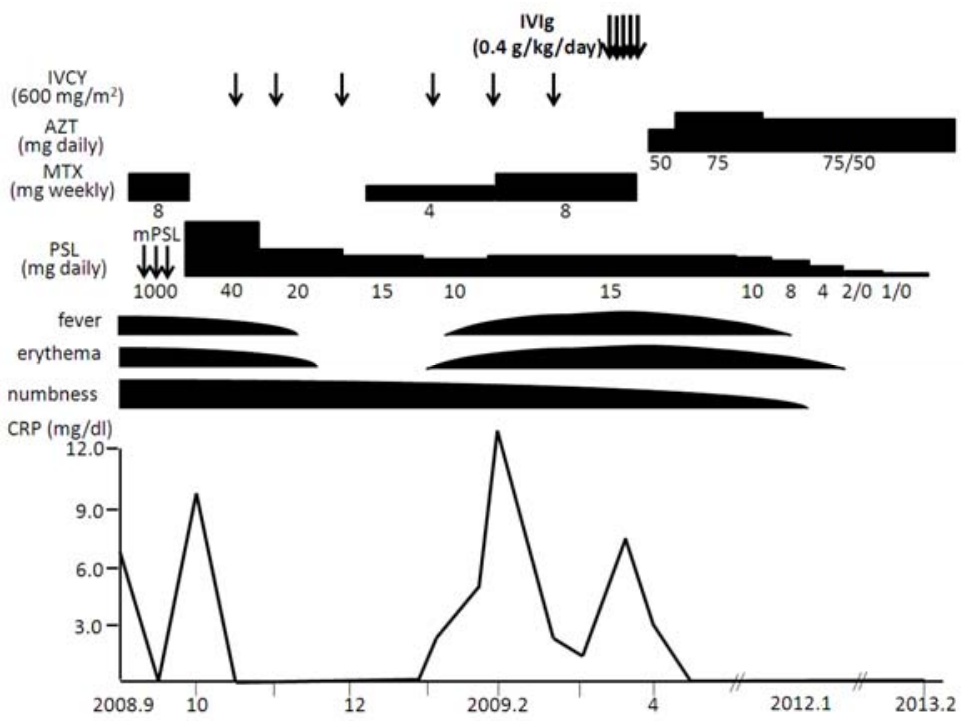

Figure 2. Clinical course. 


\section{Discussion}

PAN is a type of vasculitis affecting medium- to small-sized arteries. Recent studies estimated the prevalence as approximately 30 per one million adults in France and Sweden ${ }^{[6,7]}$.

In necrotizing systemic vasculitides associated with ANCA, IVIG therapy resulted in complete or partial responses in $45 \%-75 \%$ of patients ${ }^{[2]}$. To date, various mechanisms of action for IVIG in autoimmune disorders have been postulated, such as the blockade of $\mathrm{Fc} \gamma$ receptors on reticuloendothelial cells, alteration of $\mathrm{T}$ and/or $\mathrm{B}$ cell functions and the provision of anti-cytokine antibodies that alter immune responses and lead to the down-regulation of the immune response ${ }^{[3]}$. It was suggested that IVIG could prevent vasculitis by binding to the Fc $\gamma$ receptor, thus preventing immune complex deposition in the vessels, or by neutralization of anti-endothelial antibodies that might cause inflamed vasculitis in PAN through antiidiotypic antibodies. Thus, IVIG likely interacts with different components of the immune system, including cytokines, complement with Fc receptors and several cell surface molecules ${ }^{[2,8]}$.

It was previously reported that IVIG could be an important adjunct in selected patients with PAN, especially PAN associated with bacterial or viral infections. In such cases it led to a complete remission of the disease due to the neutralization of immune activation triggers, such as Parvovirus B19 and Streptococcus ${ }^{[9]}$. Thus, IVIG could be an excellent source of parvovirus B19-specific IGG and antibodies to streptococcal superantigens.

However, some reports have shown that the effect of IVIG therapy for PAN might be temporary ${ }^{[9,10]}$. This might be explained by the lack of combined treatment with immunosuppressants, where IVIg is used as single agent, or by early tapering of immunosuppressants. Another cause might be the dose of IVIg required for successful treatment as demonstrated by the improvement of symptoms after the initial therapy with $0.5 \mathrm{~g} / \mathrm{kg} /$ week and the recurrence of symptoms after the reduction to $0.25 \mathrm{~g} / \mathrm{kg} /$ week. Therefore, they concluded that successful treatment might correlate with the dose of IVIG per body weight and week of treatment ${ }^{[10]}$. Therefore, in the present case, we guess that high-dose and multiple courses of IVIG therapy combined with immunosuppressants could be currently maintaining a complete remission for PAN without flare-up for an additional 4 years.

In summary, the present case suggests that high-dose IVIG therapy could induce and maintain complete remission for PAN over the long-term. Therefore, high-dose IVIG therapy might be considered as a first-line therapy in patients with PAN or alternative therapy in refractory PAN.

\section{Disclosure statement}

The authors have declared no conflicts of interest.

\section{References}

[1] Guillevin L, Le Thi Huong D, Godeau P, Jais P, Wechsler B. Clinical findings and prognosis of polyarteritis nodosa and ChurgStrauss angiitis: a study in 165 patients. Br J Rheumatol. 1988; 27: 258-264. http://dx.doi.org/10.1093/rheumatology/27.4.258

[2] Martinez V, Cohen P, Pagnoux C, Vinzio S, Mahr A, Mouthon L et al. Intravenous immunoglobulins for relapses of systemic vasculitides associated with antineutrophil cytoplasmic autoantibodies: results of a multicenter, prospective, open-label study of twenty-two patients. Arthritis Rheum. 2008; 58: 308-317. http://dx.doi.org/10.1002/art.23147

[3] Ballow M. Mechanisms of action of intravenous immuneserum globulin in autoimmune and inflammatory diseases. J Allergy Clin Immunol. 1997; 100: 151-157. http://dx.doi.org/10.1016/S0091-6749(97)70217-3

[4] Levy Y, Uziel Y, Zandman G, Rotman P, Amital H, Sherer Y, Langevitz P, Goldman B, Shoenfeld Y. Response of vasculitic peripheral neuropathy to intravenous immunoglobulin. Ann N Y Acad Sci. 2005; 1051: 779-786. http://dx.doi.org/10.1196/annals.1361.121

[5] Balbir-Gurman A, Nahir AM, Braun-Moscovici Y. Intravenous immunoglobulins in polyarteritis nodosa restricted to the limbs: case reports and review of the literature. Clin Exp Rheumatol. 2007; 25: 28-30. 
[6] Mahr A, Guillevin L, Poissonnet M, Aymé S. Prevalences of polyarteritis nodosa, microscopic polyangiitis, Wegener's granulomatosis, and Churg-Strauss syndrome in a French urban multiethnic population in 2000: a capture-recapture estimate. Arthritis Rheum. 2004; 51: 92-99. http://dx.doi.org/10.1002/art.20077

[7] Mohammad AJ, Jacobsson LT, Mahr AD, Sturfelt G, Segelmark M. Prevalence of Wegener's granulomatosis, microscopic polyangiitis, polyarteritis nodosa and Churg-Strauss syndrome within a defined population in southern Sweden. Rheumatology (Oxford). 2007; 46: 1329-1337. http://dx.doi.org/10.1093/rheumatology/kem107

[8] Guillevin L. Advances in the treatments of systemic vasculitides. Clin Rev Allergy Immunol. 2008; 35: 72-78. http://dx.doi.org/10.1007/s12016-007-8068-4

[9] Asano Y, Ihn H, Maekawa T, Kadono T, Tamaki K. High-dose intravenous immunoglobulin infusion in polyarteritis nodosa: report on one case and review of the literature. Clin Rheumatol. 2006; 25: 396-398. http://dx.doi.org/10.1007/s10067-005-0015-2

[10] Kroiss M, Hohenleutner U, Gruss C, Glaessl A, Landthaler M, Stolz W. Transient and partial effect of high-dose intravenous immunoglobulin in polyarteritis nodosa. Dermatology. 2001; 203: 188-189. http://dx.doi.org/10.1159/000051741 